\title{
Editorial
}

\section{Teachers' identities under the magnifying glass in the EFL field: Crossing intellectual borders}

As a coeditor of CALJ, I would like to draw your attention to the rising importance of identity studies in the EFL setting and their contribution to the field. The Socratic imperative "know thyself" has inspired teacher researchers around the world (Benwell \& Stokoe, 2006; Cheung, 2015; Johnson \& Golombek, 2016; Norton, 2013) to raise awareness towards knowledgepower relations affecting our own constitution as subjects (Foucault, 1980). From a poststructuralist view, the comprehension of identity as something not given but constituted has illuminated a type of research more interested in revealing how interior and exterior forces -in Deleuze's (1993) words-influence our constitution as subjects of a practice. In the field of EFL, research examining identity contributes to the understanding of who English teachers and learners are and how these identities are related to the teaching and learning process.

When looking specifically at local studies, one has the sensation that a double effect has resulted from the use of identity as a category of analysis. On the one hand, its use has empowered the critical positions of researchers regarding sociocultural aspects that define and shape English teaching (Bonilla E Cruz, 2014), English teachers' roles in relation to policies and English teachers' identities (Gonzalez, 2010; Mendez, 2016; Quintero \& Guerrero, 2013), English teachers' practices of interaction (Fajardo, 2013), and English teachers' selfperception of their non-nativeness (Viáfara, 2016). On the other hand, it has increased the interest of English teachers in their students' identities not only to understand aspects affecting the learning of the target language, but also to understand how aspects of identity such as gender, age, culture, and interest might interfere with the teacher and the language per se (Castañeda-Peña, 2009).

The knowledge emerging from these revisions is relevant to informing different layers of analysis (professional, personal, academic) that mainly contribute to stand for a self- directed existence to make better decisions and to be aware of events, experiences, and practices that affect and are affected by them. I would like to remark on two important aspects: First, the regaining of status of foreign languages pedagogies in the EFL classroom. Due to reflective practices, promoted by identity research procedures, English teachers' pedagogical knowledges-obscured by dominant discourses that reify methods or approaches and give a subaltern position to pedagogy — are being valued and validated to design curricula, syllabi, and courses within a complex understanding of what is involved in teaching English as a foreign language in the expanding circle. Second, the new trends in English teacher preparation programs in which the preoccupation with education to face inequality, social justice, discrimination, and segregation has claimed teachers' who see themselves as agents of change, promoting teaching practices that contribute to solve these problems (CochranSmith, 2004; Sharkey, Clavijo Olarte, E Ramirez, 2016). From a sociocultural perspective in this reasoning, being part of an institution shapes how teachers enact their teaching and develop activities appropriated for their own purposes and understanding of the context of use, its norms, values, and opportunities for growth and development (Johnson E Golombek, 2011, 2016).

Teachers' pedagogical knowledge and, most importantly, teachers' knowledge of their sociocultural context are paramount to teaching English with political stances towards dominant discourses that determine what and how to teach as well as assuming the debate surrounding bilingual education. This means the adoption of a critical position that problematizes how "the must-be discourses" can be resisted, adapted, or re-elaborated to 
devise local relevant pedagogies and revealing how our identity is constituted in relations to students, knowledge, institutions, and practices. As a way to exemplify, studies examining students' identities in the EFL field have helped teachers to make informed decisions and understand very important practices as the role of students' interaction into the classroom in located teaching setting (Benavides, this volume; Sharkey, Clavijo-Olarte, \& Ramirez 2016). Here, the magnifying glass focuses on students' language used as a means to negotiate (affirm, modify, approve, or disapprove of) meaning and self-knowledge that might unveil how relations of power affect learners.

From my own work as teacher-researcher on the teacher-subject constitution complexity, being seduced-epistemologically speaking-by philosophical and decolonial dissertations within an interdisciplinary framework has been an open invitation to value local practices, teachers and students' voices, and to understand the complex process of becoming who we are by means of reflecting upon how we struggle to be the teachers we are. I would like to extend this invitation to all teacher-researchers to get in singularities, forms of mutual understanding.

\section{References}

Benavides, C. (2017). EFL students' social identities construction through gender-based short stories. Colombian Applied Linguistics Journal, 19(1), (11-21)

Benwell, B., \& Stokoe, E. (2006). Discourse and identity. Edinburgh, UK: Edinburgh University Press

Bonilla, S. X., \& Cruz, F. (2014). Critical socio-cultural elements of the intercultural endeavour of English teaching in Colombian rural areas. PROFILE Journal, 16(2), 117-133.

Castañeda-Peña, H. (2009). Masculinities and femininities go to preschool: Gender positioning in discourse. Bogotá, Colombia: Editorial Pontificia Universidad Javeriana.

Cochran-Smith, M (2004). Walking the road: Race, diversity, and social justice in teacher education. New York, NY: Teachers College Press.

Cheung, Y. (2015). Teacher identity in EFL/TESOL: A research review. In Y. Ling, S. Ben, E P. Kwanghyun (Eds.). Advances and current trends in language teacher identity research (pp. 175-185). London: Routledge.

Deleuze, G. (1993). The fold: Leibniz and the Baroque (Tom Conley, Trans.). Minneapolis: University of Minnesota Press.

Fajardo Castañeda, J. A. (2013). "What Makes a Teacher": Identity and classroom talk. Cuadernos de Lingüística Hispánica, 1(22), 127-146. Retrieved from: http://www.redalyc. org/articulo.oa?id=322229879009

Foucault, M. (1980). Power-knowledge. Brighton: Harvester

González, A. (2010). English and English teaching in Colombia: Tensions and possibilities in the expanding circle. In A. Kirkpatrick (Ed.), The Routledge handbook of world Englishes (pp. 332-351). London: Routledge.

Johnson, K. E., \& Golombek, P. (2011). Research on second lanquage teacher education: A sociocultural perspective on professional development. New York, NY: Routledge.

Johnson, K, E Golombek, P. (2016). Mindful L2 teacher education: A sociocultural perspective on cultivating teachers' professional development. New York, NY: Routledge.

Méndez, P. (2016). Voces de los profesores de inglés sobre Bogotá bilingüe. Ponencia Congreso USTA. Bogotá. Disponible en: http://soda.ustadistancia.edu.co/enlinea/congreso/ congresoedu/3\%20Politicas\%20educativas $\% 20$ y\%20derechos $\% 20$ humanos/3\%20 21\%20VOCES\%20DE\%20LOS\%20PROFESORES\%20DE\%20INGLES\%20SOBRE\%20 BOGOTA\%20BILINGUE.pdf

Norton, B. (2013). Identity and language learning: Extending the conversation. Clevedon: Multilingual Matters.

Quintero, A. H., \& Guerrero, C; H. (2013). Of being and not being: Colombian public elementary school teachers' oscillating identities. HOW: A Colombian Journal for Teachers of English, 20(1), 190-205.

Sharkey, J., Clavijo, A., E Ramírez, M. (2016) Developing a deeper understanding of communitybased pedagogies with teachers: Learning with and from teachers in Colombia. Journal of Teacher Education 67(3), 1-14.

Viáfara, J. J. (2016). "I'm Missing Something": (Non) nativeness in prospective teachers as Spanish and English speakers. Colombian Applied Linguistics Journal, 18(2), 11-24.

Pilar Méndez PhD

Amparo Clavijo PhD

Editors 


\section{Editorial}

\section{Identidad de los profesores bajo la lupa en el campo de la Enseñanza del Inglés como Lengua Extranjera: El cruce de fronteras intelectuales}

Como coeditora de la revista Colombian Applied Linguistics, me gustaría llamar la atención
hacia la importancia creciente de estudios de identidad en la Enseñanza del Inglés como
Lengua Extranjera y su contribución al campo. El imperativo socrático "Conócete a ti mismo"
ha inspirado a los investigadores docentes de todo el mundo (Benwell E Stokoe, 2006;
Cheung, 2015; Johnson \& Golombek, 2016; Norton, 2013) a incrementar la conciencia hacia
relaciones de poder-saber que afectan nuestra propia constitución como sujetos (Foucault,
1980). Desde una perspectiva post-estructuralista, la comprensión de la identidad como algo
no dado sino constituido, ha iluminado un tipo de investigación más interesada en revelar
cómo las fuerzas interiores y exteriores -en palabras de Deleuze (1993)-influyen en nuestra
constitución como sujetos de una práctica. En el campo de la Enseñanza del Inglés como
o lengua extranjera, la investigación que examina la identidad contribuye a la comprensión
de quiénes son los profesores y los estudiantes de inglés y cómo estas identidades están
relacionadas con el proceso de enseñanza y aprendizaje. Al mirar específicamente los estudios locales, uno tiene la sensación de que un doble efecto ha resultado del uso de la identidad como una categoría de análisis. Por un lado, su uso ha potenciado las posiciones críticas de los investigadores respecto a los aspectos socioculturales que definen y modelan la enseñanza del inglés (Bonilla y Cruz, 2014), los roles de los profesores de inglés en relación con las políticas y las identidades de los mismos (González, 2010; Méndez, 2016; Quintero y Guerrero, 2013), las prácticas de interacción de los profesores de inglés (Fajardo, 2013) y la autopercepción de los profesores de inglés de su no-natividad (Viáfara, 2016). Por otra parte, ha aumentado el interés de los profesores de inglés por las identidades de sus estudiantes no sólo para comprender aspectos que afectan al aprendizaje de la lengua objetivo, sino también para entender cómo aspectos de la identidad como el género, la edad, la cultura y el interés pueden interferir con el profesor y el lenguaje en sí (Castañeda-Peña, 2009).

El conocimiento que surge de estas revisiones es relevante para informar a diferentes capas de análisis (profesional, personal, académica) que contribuyen principalmente a defender una existencia auto dirigida para tomar mejores decisiones y ser conscientes de los acontecimientos, experiencias y prácticas que afectan y son afectados por ellos. Me gustaría resaltar dos aspectos importantes: Primero, la recuperación del estatus de las pedagogías de lenguas extranjeras en el aula. Debido a las prácticas reflexivas promovidas por los procedimientos de investigación de la identidad, los conocimientos pedagógicos de los profesores de inglés, oscurecidos por discursos dominantes que reifican métodos o enfoques y dan una posición subalterna a la pedagogía, están siendo valorados y validados para diseñar planes de estudio y cursos dentro de un complejo entendimiento de lo que implica la enseñanza del inglés como lengua extranjera en el círculo en expansión. En segundo lugar, las nuevas tendencias en los programas de preparación de profesores de inglés en los que la preocupación por la educación frente a la desigualdad, la justicia social, la discriminación y la segregación ha reclamado a los maestros que se vean a sí mismos como agentes de cambio, promoviendo prácticas docentes que contribuyen a resolver estos problemas (Cochran-Smith, 2004; Sharkey, Clavijo- Olarte, E Ramirez, 2016). Desde una perspectiva sociocultural en este razonamiento, formar parte de una institución moldea cómo los docentes promulgan su enseñanza y desarrollan actividades apropiadas para sus propios propósitos y comprensión del contexto de uso, sus normas, valoresy las oportunidades de crecimiento y desarrollo (Johnson \& Golombek , 2011, 2016).

El conocimiento pedagógico de los maestros y, lo que es más importante, el conocimiento de los maestros de su contexto sociocultural son fundamentales para enseñar inglés con 
posiciones políticas hacia discursos dominantes que determinan qué y cómo enseñar y asumir el debate alrededor de la educación bilingüe. Esto significa la adopción de una posición crítica que problematiza cómo se pueden resistir, adaptar o reelaborar los "discursos del deber ser" para idear las pedagogías pertinentes locales y revelar cómo se constituye nuestra identidad en las relaciones con los estudiantes, el conocimiento, las instituciones y las prácticas. Como una manera de ejemplificar, los estudios que examinan las identidades de los estudiantes en el campo de la Enseñanza del Inglés como lengua Extranjera han ayudado a los maestros a tomar decisiones informadas y entender prácticas muy importantes como lo es el papel de la interacción de los estudiantes en el aula en un ambiente de enseñanza localizado (Benavides, 2017; Sharkey, Clavijo Olarte, E Ramírez 2016). Aquí, la lupa se centra en el lenguaje de los estudiantes utilizado como un medio para negociar (afirmar, modificar, aprobar o desaprobar) el significado y el autoconocimiento que podría revelar cómo las relaciones del poder afectan a los estudiantes.

Desde mi propio trabajo como docente-investigadora sobre la complejidad de la constitución maestro-sujeto, seducida -epistemológicamente hablando- por disertaciones filosóficas y descoloniales, dentro de un marco interdisciplinario, ha sido una invitación abierta a valorar las prácticas locales, las voces de profesores y estudiantes y, comprender el proceso complejo de convertirnos en quienes somos mediante la reflexión sobre cómo luchamos por ser los maestros que somos. Quisiera extender esta invitación a todos los docentes-investigadores para encontrar en las singularidades, formas de entendimiento mutuo.

\section{Referencias}

Benavides, C. (2017). EFL students' social identities construction through gender-based short stories. Colombian Applied Linguistics Journal, 19(1), (11-21)

Benwell, B., \& Stokoe, E. (2006). Discourse and identity. Edinburgh, UK: Edinburgh University

Press
Bonilla, S. X., \& Cruz, F. (2014). Critical socio-cultural elements of the intercultural endeavour of English teaching in Colombian rural areas. PROFILE Journal, 16(2), 117-133.

Castañeda-Peña, H. (2009). Masculinities and femininities go to preschool: Gender positioning in discourse. Bogotá, Colombia: Editorial Pontificia Universidad Javeriana.

Cochran-Smith, M (2004). Walking the road: Race, diversity, and social justice in teacher education. New York, NY: Teachers College Press.

Cheung, Y. (2015). Teacher identity in EFL/TESOL: A research review. In Y. Ling, S. Ben, E P. Kwanghyun (Eds.). Advances and current trends in language teacher identity research (pp. 175-185). London: Routledge.

Deleuze, G. (1993). The fold: Leibniz and the Baroque (Tom Conley, Trans.). Minneapolis: University of Minnesota Press.

Fajardo Castañeda, J. A. (2013). "What Makes a Teacher": Identity and classroom talk Cuadernos de Lingüística Hispánica, 1(22), 127-146. Retrieved from: http://www.redalyc. org/articulo.oa?id=322229879009

Foucault, M. (1980). Power-knowledge. Brighton: Harvester.

González, A. (2010). English and English teaching in Colombia: Tensions and possibilities in the expanding circle. In A. Kirkpatrick (Ed.), The Routledge handbook of world Englishes (pp. 332-351). London: Routledge.

Johnson, K. E., \& Golombek, P. (2011). Research on second language teacher education: A sociocultural perspective on professional development. New York, NY: Routledge.

Johnson, K, E Golombek, P. (2016). Mindful L2 teacher education: A sociocultural perspective on cultivating teachers' professional development. New York, NY: Routledge.

Méndez, P. (2016). Voces de los profesores de inglés sobre Bogotá bilingüe. Ponencia Congreso USTA. Bogotá. Disponible en: http://soda.ustadistancia.edu.co/enlinea/congreso/ congresoedu/3\%20Politicas\%20educativas $\% 20$ y 20 derechos $\% 20$ humanos $/ 3 \% 20$ 21\%20VOCES\%20DE\%20LOS\%20PROFESORES\%20DE\%20INGLES\%20SOBRE\%20 BOGOTA\%20BILINGUE.pdf

Norton, B. (2013). Identity and language learning: Extending the conversation. Clevedon: Multilingual Matters.

Quintero, A. H., \& Guerrero, C. H. (2013). Of being and not being: Colombian public elementary school teachers' oscillating identities. HOW: A Colombian Journal for Teachers of English, 20(1), 190-205.

Sharkey, J., Clavijo, A., E Ramírez, M. (2016) Developing a deeper understanding of communitybased pedagogies with teachers: Learning with and from teachers in Colombia. Journal of Teacher Education 67(3), 1-14.

Viáfara, J. J. (2016). "I'm Missing Something": (Non) nativeness in prospective teachers as Spanish and English speakers. Colombian Applied Linguistics Journal, 18(2), 11-24.

Pilar Méndez PhD

Amparo Clavijo PhD

Editoras 